\title{
Circulating omentin-1 levels and inflammation in polycystic ovary syndrome
}

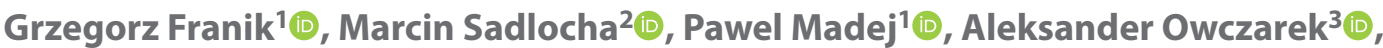

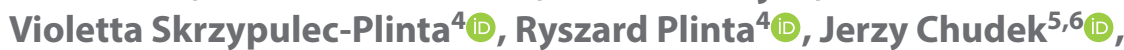 \\ Magdalena Olszanecka-Glinianowicz ${ }^{7}$ (])
}

\author{
${ }^{1}$ Department of Endocrinological Gynecology, Faculty of Medical Sciences in Katowice, Medical University of Silesia, Katowice, Poland \\ ${ }^{2}$ Women's Health Chair, School of Health Science in Katowice, Medical University of Silesia, Katowice, Poland \\ ${ }^{3}$ Department of Statistics, Faculty of Pharmaceutical Sciences in Sosnowiec, Medical University of Silesia, Katowice, Poland \\ ${ }^{4}$ Chair of Physiotherapy, School of Health Science in Katowice, Medical University of Silesia, Katowice, Poland \\ ${ }^{5}$ Pathophysiology Unit, Department of Pathophysiology, Faculty of Medical Sciences in Katowice, \\ Medical University of Silesia, Katowice, Poland \\ ${ }^{6}$ Department of Internal Medicine and Oncological Chemotherapy, Faculty of Medical Sciences in Katowice, \\ Medical University of Silesia, Katowice, Poland \\ ${ }^{7}$ Health Promotion and Obesity Management Unit, Department of Pathophysiology, \\ Faculty of Medical Sciences in Katowice, Medical University of Silesia, Katowice, Poland
}

\begin{abstract}
Objectives: The aim of the study was to analyze interrelation between plasma omentin- 1 levels and nutritional status and inflammation in PCOS.

Material and methods: A cross-sectional study involving 86 PCOS (47 obese) and 72 Non-PCOS women (41 obese) determined anthropometric parameters and body composition. Serum glucose, insulin and omentin-1, TNF-a, sTNFRs, IL-6 and sR-IL6 were measured in the fasting state.

Results: Plasma omentin-1 levels were significantly lower in the PCOS than in the Non-PCOS group and both corresponding normal weight and obese subgroups. In three analyzed least-angle regression (LARS) models the lower plasma omentin-1 levels was associated with PCOS occurrence, higher circulating TNF- $a$ and lower IL-6 levels.

Conclusions: Suppressed omentin-1 levels in PCOS are characteristic for this disturbance and proinflammatory cytokines are factors modifying secretion of this adipokine.
\end{abstract}

Key words: omentin-1; inflammation; nutritional status; PCOS

Ginekologia Polska 2020; 91, 6: 308-312

\section{INTRODUCTION}

Omentin-1 is an adipokine, released mainly by stromal-vascular cells of adipose tissue [1-3]. The highest omentin mRNA expression was shown in visceral and pericardial adipose tissues, while in subcutaneous is twenty times lower [3].

It has been shown in an experimental model that omentin-1 stimulates Akt phosphorylation and increases insulin-stimulated glucose uptake [2]. In addition, omentin-1 exerts anti-inflammatory properties, and is also associated with decreased cardiovascular risk [4]. Notably, circulating omentin-1 levels are higher in women than in men [5].
Decreased expression, secretion and circulating levels of omentin-1 were shown in obese subjects [5]. Moreover, negative correlations between omentin-1 concentrations and $\mathrm{BMI}$, waist circumference, circulating leptin and insulin levels and positive with adiponectin levels were observed [5]. In addition, an inhibiting effect of high CRP levels on omentin-1 concentrations was found [6]. It has also been shown that weight loss is followed by omentin-1 level increase, proportional to the improvement in insulin sensitivity accompanied by a decrease of insulin levels [7]. Furthermore, 12 -weeks of aerobic training causing decreased fat depots 
without changes in body mass was followed by an increase in circulating omentin-1 levels [8].

The results of studies assessing omentin-1 levels in PCOS are inconsistent. Its lower circulating levels were shown independent from nutritional status and inversely associated with androgens and CRP levels [9]. While, no difference between normal weight PCOS and Non-PCOS women was shown despite higher insulin levels and marked insulin resistance in PCOS group [10]. Contrary, Than et al. [6] observed that metformin therapy increased omentin-1 levels in PCOS women.

Recently, we have suggested an impairment of hormonal stroma adipose tissue function regardless of the nutritional status, secondary to insulin resistance and hyperandrogenism in PCOS [11]. We have also found that low grade inflammation is related to nutritional status, independently from PCOS occurrence [12]. So far, only one study documented negative correlation between levels of omentin-1 and IL- 6 and TNF- $a$ in PCOS women in the Asian group [13].

The aim of the study was to analyze interrelation between plasma omentin- 1 levels and nutritional status and inflammation in PCOS women.

\section{MATERIAL AND METHODS}

The cross-sectional study involved 86 PCOS women (39 normal weight and 47 obese) with stable body mass during last 3-month period diagnosed in Department of Endocrinological Gynecology from 2013 to 2014. The diagnosis of PCOS was based on Rotterdam ESHRE/ASRM criteria from 2003 [14]. Seventy-two women without PCOS (31 normal weight and 41 obese) constitute the control group. Patients with Cushing's syndrome, thyroid dysfunctions, androgen secreting tumor, and enzyme deficiency (21-hydroxylase in particular), decreased ovary reserves, type 1 and 2 diabetes were not enrolled. Any pharmacological therapy, smoking and alcohol abuse were among the exclusion criteria. The study was conducted after obtaining of the informed consent from each participant. Study protocol was approved by the Ethical Committee of Medical University of Silesia.

Normal weight was defined as body mass index (BMI) from 18.5 to $24.9 \mathrm{~kg} / \mathrm{m}^{2}$ and obesity as $\geq 30.0 \mathrm{~kg} / \mathrm{m}^{2}$. The characteristics of the study groups are presented in Table 1.

All the study women were tested within 3 and 5 days of menstrual cycle. Anthropometric measurements (body mass, height and waist circumference) were performed, and BMI was calculated according to the standard formula. Body composition was assessed by bioimpedance method using Bodystat 1500 (Douglas, Isle of Man). $15 \mathrm{~mL}$ samples of venous blood were withdrawn in the morning between 8.00-9.00 a.m., after an overnight fast (16 h). The blood samples were collected according to recommendation of manufacturer of the kits. Serum and plasma samples were stored frozen in $-70^{\circ} \mathrm{C}$.

\section{Laboratory procedures}

Plasma glucose was estimated by colorimetric methods using the commercially available test kits (Roche, Switzerland). Serum insulin concentration was determined by enzyme-linked immunosorbent assay (ELISA) (DRG Instruments $\mathrm{GmbH}$, Marburg, Germany) with a lower limit of sensitivity of $1.76 \mu \mathrm{lU} / \mathrm{mL}$ and intra- and inter-assay coefficients of variations of $2.2 \%$ and $4.4 \%$, respectively. HOMA-IR index was calculated with the standard formula: $\mathrm{HOMA}-\mathrm{IR}=$ fasting concentration of insulin $(\mu \mathrm{lU} / \mathrm{mL}) \times$ fasting concentration of glucose $(\mathrm{mmol} / \mathrm{L}) / 22.5$.

ELISA method was also used for measurements of plasma omentin-1 levels (BioVendor, Brno, Czech Republic) with the lower limit of sensitivity of $0.5 \mathrm{ng} / \mathrm{mL}$ and intra- and inter-assay coefficients of variations were $3.65 \%$ and $4.6 \%$, respectively as well as TNF-a, sTNFR1, sTNFR2, IL-6 and sR-IL-6 (R\&D Systems, Michigan, USA) with the lower limit of sensitivity $0.18 \mathrm{pg} / \mathrm{mL}, 0.77 \mathrm{pg} / \mathrm{mL}, 0.6 \mathrm{pg} / \mathrm{mL}, 0.5 \mathrm{pg} / \mathrm{mL}$ and $0.88 \mathrm{pg} / \mathrm{mL}$, respectively and intra- and inter-assay coefficients of variations were $14.4 \%$ and $18.7 \%$ respectively for TNF-a, 3.6\% and $2.6 \%$ respectively for sTNFR1, 3.7\% and $3.5 \%$ respectively for sTNFR2, $16.8 \%$ and $17.2 \%$ respectively for IL-6 and 9.7\% and 9.6\% respectively for SR-IL-6.

\section{Statistical analysis}

Statistical analyses were performed using STATISTICA 9.0 PL (StatSoft Poland) software and R software environment. There was no missing data in the database. The results are presented as mean values \pm standard deviation. Distribution of variables was evaluated by the D'Agostino-Pearson test. Homogeneity of variances was assessed by the Levene test. Quantitative variables were compared with two-way multivariate analysis of variances with Duncan test post-hoc. The assessment of association between variables was done with the multivariate linear regression and the backward stepwise procedure. Outliers were identified based on Cook's distance values. The Cook-Weisberg test was used to test the residuals for heteroskedasticity. Models calculation was performed including evaluation of multicollinearity, which was assessed with the variance inflation factor (VIF). The VIF should not exceed more than five. Goodness of fit of obtained model was assessed with the $F$ test and determination coefficient $R^{2}$. All results were considered as statistically significant with a $p$ value of $<0.05$.

\section{RESULTS}

The age of both obese subgroups (PCOS and Non-PCOS) as well as of normal weight subgroups (PCOS and Non-PCOS) 


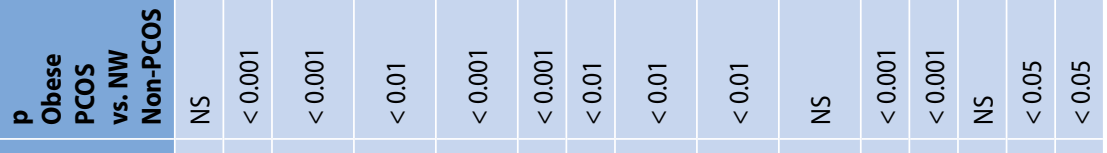

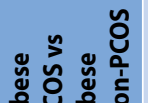

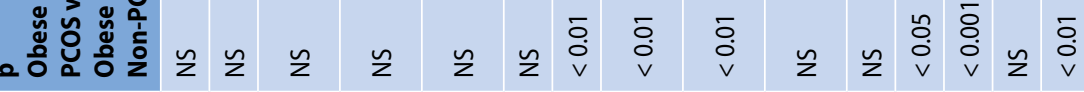

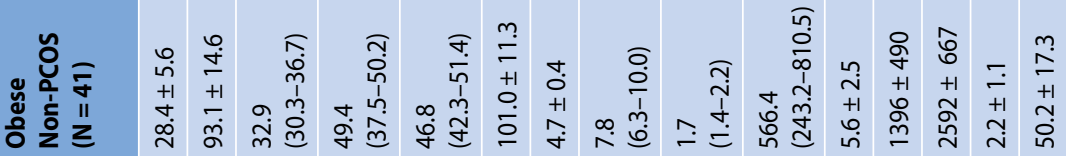

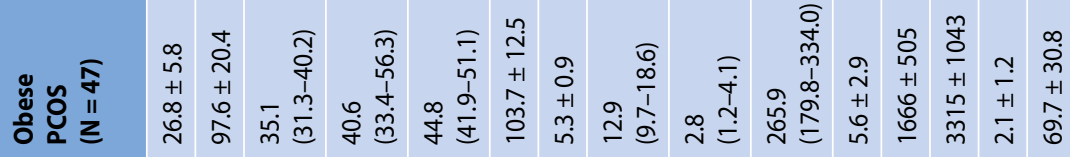

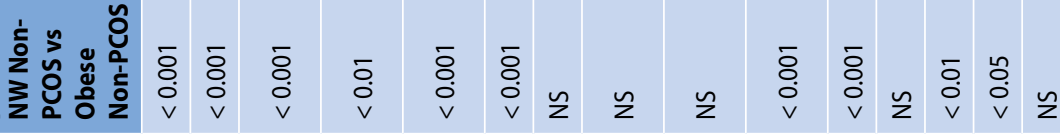

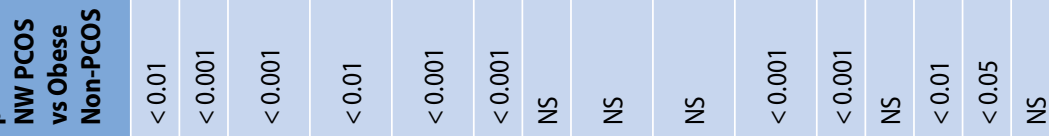

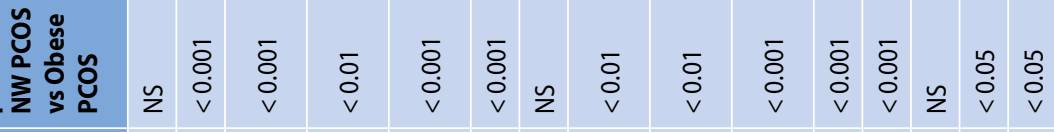

萬莒

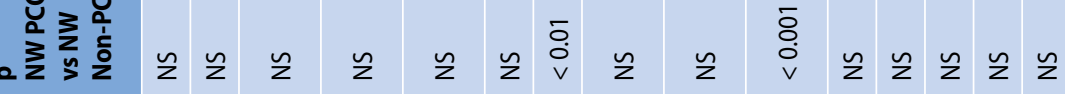

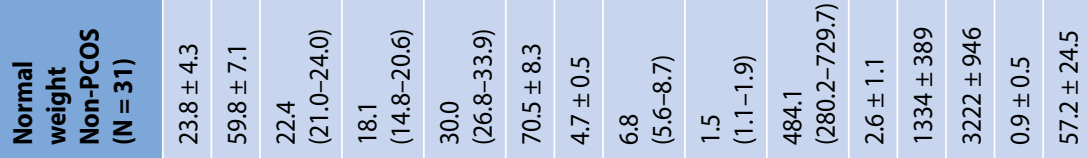

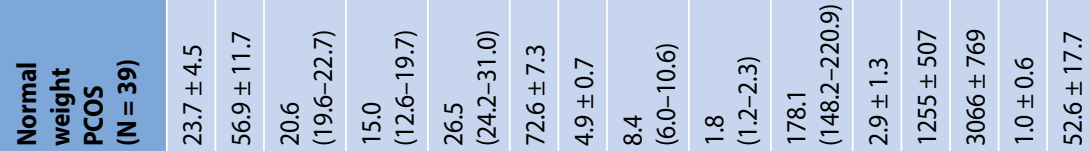
药

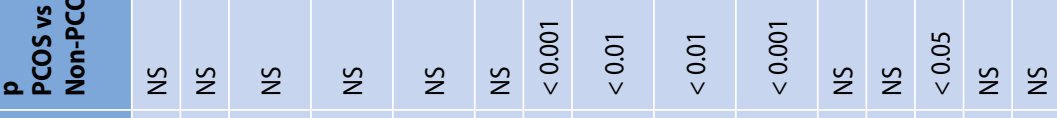

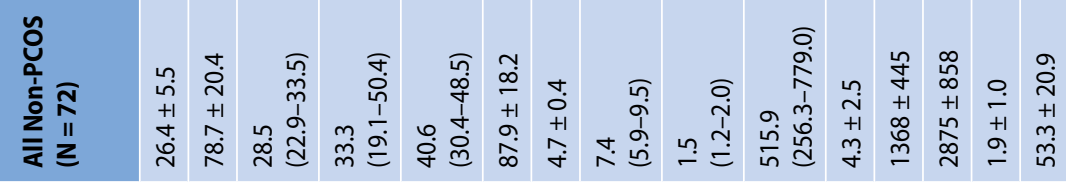

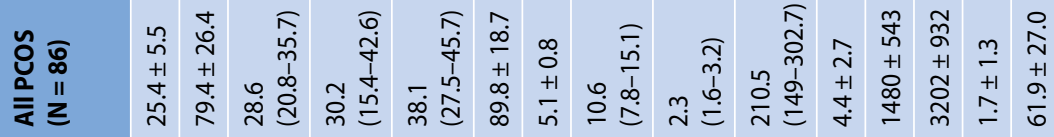

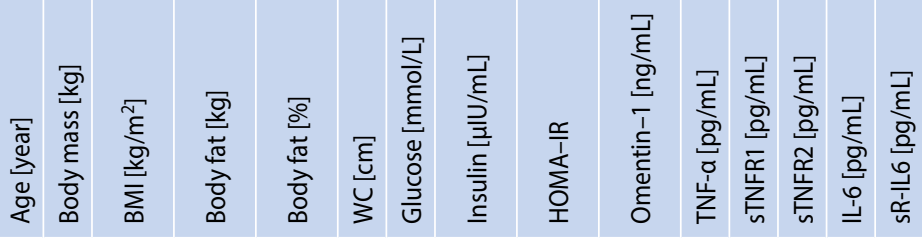


was similar (Tab. 1). Body mass and BMI did not differ between the corresponding subgroups of PCOS and Non-PCOS.

The serum concentrations of glucose, insulin and HOMA-IR values were significantly higher in the PCOS group than in the Non-PCOS group. As expected, in the obese PCOS subgroup, serum concentration of insulin and HOMA-IR value were significantly higher than in normal weight PCOS subgroup (Tab. 1).

Plasma omentin-1 levels were significantly lower in the PCOS group than in the Non-PCOS group. The lower plasma omenntin-1 levels were shown in normal weight and obese PCOS than in both corresponding Non-PCOS subgroups (Tab. 1).

There were no differences in plasma TNF- $a$,TNFR1, IL- 6 and sR-IL- 6 levels between the PCOS and Non-PCOS groups, while TNFR2 levels were significantly higher in the PCOS group. However, plasma TNF- $a$ and IL-6 levels were significantly higher in both obese PCOS and Non-POS than in corresponding normal weight subgroups. The highest sTNFR1 levels were observed in the obese PCOS subgroup. While, sTNFR2 and SR-IL-6 levels were significantly higher in obese than normal weight PCOS subgroups and significantly lower in obese than normal weight Non-PCOS subgroups (Tab. 1).

\section{Multiple regression analyses}

In Table 2, presented are three analyzed Least-angle regression (LARS) models. Numbers denote sequence of switching on of the variable model. The best results, the lowest fault model fit to the data, in each case, was obtained for a set of three variables. The lower plasma omentin-1 levels were associated with PCOS occurrence, higher circulating TNF- $a$ and lower IL-6 levels (Tab. 2).

\section{DISCUSSION}

Numerous studies published in recent years, including ours, suggested that hormonal dysfunction of adipose tis-

Table 2. The models regression type LARS and the resulting model of the impact of variable on plasma omentin-1 levels

\begin{tabular}{|l|l|l|l|l|}
\hline $\begin{array}{l}\text { Variable } \\
\mathbf{y}=\log _{10}(\text { Omentin-1) }[\mathrm{ng} / \mathrm{mL}]\end{array}$ & $\begin{array}{l}\text { Model } \\
\text { I }\end{array}$ & $\begin{array}{l}\text { Model } \\
\text { II }\end{array}$ & $\begin{array}{l}\text { Model } \\
\text { III }\end{array}$ & $\begin{array}{l}\text { Final } \\
\text { model }\end{array}$ \\
\hline PCOS \pm & 1 & 1 & 1 & -0.2432 \\
\hline BMI [kg/m²] & 8 & - & - & - \\
\hline Fat percentage [\%] & - & 8 & - & - \\
\hline Waist circumference [cm] & - & - & 8 & - \\
\hline $\log _{10}($ HOMA-IR) & 7 & 7 & 7 & - \\
\hline $\log _{10}($ TNF-a) $[\mathrm{pg} / \mathrm{mL}]$ & 3 & 3 & 3 & -0.0652 \\
\hline $\log _{10}(\mathrm{sTNF}-\mathrm{R} 1)[\mathrm{pg} / \mathrm{mL}]$ & 5 & 5 & 5 & - \\
\hline sTNF-R2 [pg/mL] & 6 & 6 & 6 & - \\
\hline $\log _{10}($ IL-6) $[\mathrm{pg} / \mathrm{mL}]$ & 2 & 2 & 2 & 0.0745 \\
\hline $\log _{10}(\mathrm{sR}-\mathrm{IL} 6)[\mathrm{pg} / \mathrm{mL}]$ & 4 & 4 & 4 & - \\
\hline
\end{tabular}

sue is an important link in PCOS pathogenesis [11, 15-18]. Obesity, especially visceral, is well known risk factor of PCOS development in genetically predisposed women [19, 20]. Inflammation in visceral adipose tissue is a key factor affecting the release of adipokines [21]. The changes in the profile of circulating adipokines were shown to be associated with the development of insulin resistance and both pituitary and ovary hormonal dysfunction [22].

The previously published studies have shown lower plasma omentin-1 levels in PCOS women, independently from nutritional status $[6,9,11]$. Contrary to some studies $[5,10]$ we did not observe the association between plasma omentin-1 levels and anthropometric parameters. This discrepancy is difficult to explain. However, the results of our recently published study suggest that hormonal dysfunction of stromal cells of adipose tissue is the result, but not a cause of insulin resistance development [11]. This hypothesis is supported by the fact that HOMA-IR values in the obese non-PCOS subgroup were below the cut-off point for the insulin resistance. Moreover, Cai et al. [23] revealed that the impaired function of stromal cells of adipose tissue is strongly associated with insulin resistance. This may explain lower omentin-1 levels in the obese, but not in the normal weight PCOS subgroup. Based on our own and other studies $[9,11]$ we suggest that androgens may be an important factor in explaining disturbances of hormonal function of stromal cells of adipose tissue. Disturbances of omentin-1 synthesis in adipose tissue are not the primary effect of visceral fat accumulation, but rather secondary to the development of insulin resistance and hormonal disturbances [11]. However, inflammation in visceral adipose tissue may be an important cause for decreased omentin- 1 synthesis. As it was mentioned above, a single study performed in an Asian PCOS group showed a negative correlation between omentin- 1 and IL- 6 and TNF- $\alpha$ levels [13]. In accordance with this data, multiple regression analysis in the current study showed a negative impact of TNF-a on omentin-1 levels. However, this association is controversial, as TNF-a levels were significantly lower in the normal weight PCOS group than both obese PCOS and Non-PCOS subgroups. In addition, TNF-a levels were similar in normal weight PCOS and Non-PCOS subgroups. These results are in accordance with our study published over 10 years ago that PCOS is not associated with chronic inflammation per se [24]. Contrary to previously published data, our study regression model suggests that IL- 6 may stimulate omentin- 1 synthesis. This discrepancy is difficult to explain, but potentially related to the dual, proinflammatory and anti-inflammatory function of IL-6. It should be noted that the proinflammatory properties of cytokines do not correspond to lower circulating omentin-1 levels in normal weigh and obese PCOS showing expected differences in TNF- $a$ and IL-6 levels $[12,25]$. Our study suggests that the mechanism of stromal cells dysfunc- 
tion in adipose tissue in PCOS is more complex, including impact of insulin resistance, androgens and proinflammatory cytokines. However, these factors may explain lower omentin-1 levels in obese PCOS, it does not fully elucidate the cause of its decreased release in normal weight PCOS. Thus, the relationship between this cytokine and omentin-1 levels requires experimental studies on cell cultures assessing the effect of this cytokine on omentin-1 expression. Although, it cannot be excluded that the decreased concentration of circulating omentin-1 in PCOS women with normal body mass is the result of its' disturbed secretion in ovaries, but not in adipose tissue [26]. Verification of this hypothesis also requires further experimental studies.

The limitation of our study is the size of the study subgroups and the lack of separation of PCOS with normal weight for subgroups with and without metabolic obesity. Moreover, the distribution of body fat and its visceral deposits were not directly assessed using DEXA or CT scanner. Additionally, in our study, the impact of selected adipokines on omentin-1 levels has not been analyzed.

\section{CONCLUSIONS}

Suppressed omentin-1 levels in PCOS are characteristic for this disturbance whereas proinflammatory cytokines are factors modifying secretion of this adipokine.

\section{REFERENCES}

1. Schäffler A, Neumeier $M$, Herfarth $H$, et al. Genomic structure of human omentin, a new adipocytokine expressed in omental adipose tissue. Biochim Biophys Acta. 2005; 1732(1-3): 96-102, doi: 10.1016/j. bbaexp.2005.11.005, indexed in Pubmed: 16386808.

2. Yang RZ, Lee $\mathrm{MJ}, \mathrm{Hu} \mathrm{H}$, et al. Identification of omentin as a novel depot-specific adipokine in human adipose tissue: possible role in modulating insulin action. Am J Physiol Endocrinol Metab. 2006; 290(6): E1253-E1261, doi: 10.1152/ajpendo.00572.2004, indexed in Pubmed: 16531507.

3. Fain JN, Sacks HS, Buehrer B, et al. Identification of omentin mRNA in human epicardial adipose tissue: comparison to omentin in subcutaneous, internal mammary artery periadventitial and visceral abdominal depots. Int J Obes (Lond). 2008; 32(5): 810-815, doi: 10.1038/sj.ijo.0803790, indexed in Pubmed: 18180782.

4. Yamawaki H, Kuramoto J, Kameshima S, et al. Omentin, a novel adipocytokine inhibits TNF-induced vascular inflammation in human endothelial cells. Biochem Biophys Res Commun. 2011; 408(2): 339-343, doi: 10.1016/j.bbrc.2011.04.039, indexed in Pubmed: 21514279.

5. de Souza Batista CM, Yang RZ, Lee MJ, et al. Omentin plasma levels and gene expression are decreased in obesity. Diabetes. 2007; 56(6): 1655-1661, doi: 10.2337/db06-1506, indexed in Pubmed: 17329619.

6. Tan BK, Adya R, Farhatullah S, et al. Metformin treatment may increase omentin-1 levels in women with polycystic ovary syndrome. Diabetes. 2010; 59(12): 3023-3031, doi: 10.2337/db10-0124, indexed in Pubmed: 20852028.

7. Moreno-Navarrete JM, Catalán V, Ortega F, et al. Circulating omentin concentration increases after weight loss. Nutr Metab (Lond). 2010; 7: 27, doi: 10.1186/1743-7075-7-27, indexed in Pubmed: 20380714.

8. Saremi A, Asghari M, Ghorbani A. Effects of aerobic training on serum omentin-1 and cardiometabolic risk factors in overweight and obese men. J Sports Sci. 2010; 28(9): 993-998, doi: 10.1080/02640414.2010.484070, indexed in Pubmed: 20544489.

9. Choi JH, Rhee EJ, Kim KH, et al. Plasma omentin-1 levels are reduced in non-obese women with normal glucose tolerance and polycystic ovary syndrome. Eur J Endocrinol. 2011; 165(5): 789-796, doi: 10.1530/EJE-110375, indexed in Pubmed: 21865408.

10. Akbarzadeh $\mathrm{S}$, Ghasemi $\mathrm{S}$, Kalantarhormozi $\mathrm{M}$, et al. Relationship among plasma adipokines, insulin and androgens level as well as biochemical glycemic and lipidemic markers with incidence of PCOS in women with normal BMI. Gynecol Endocrinol. 2012; 28(7): 521-524, doi: 10.3109/09513590.2011.650747, indexed in Pubmed: 22309615.

11. Orlik B, Madej P, Owczarek A, et al. Plasma omentin and adiponectin levels as markers of adipose tissue dysfunction in normal weight and obese women with polycystic ovary syndrome. Clin Endocrinol (Oxf). 2014 81(4): 529-535, doi: 10.1111/cen.12381, indexed in Pubmed: 24392647.

12. Olszanecka-Glinianowicz M, Banaś $M$, Zahorska-Markiewicz B, et al. Is the polycystic ovary syndrome associated with chronic inflammation per se? Eur J Obstet Gynecol Reprod Biol. 2007; 133(2): 197-202, doi: 10.1016/j. ejogrb.2006.10.037, indexed in Pubmed: 17224231.

13. Mahde A, Shaker M, Al-Mashhadani Z. Study of Omentin1 and Other Adipokines and Hormones in PCOS Patients. Oman Med J. 2009; 24(2): 108-118, doi: 10.5001/omj.2009.25, indexed in Pubmed: 22334855.

14. Rotterdam ESHRE/ASRM-Sponsored PCOS Consensus Workshop Group, Rotterdam ESHRE/ASRM-Sponsored PCOS consensus workshop group. Revised 2003 consensus on diagnostic criteria and long-term health risks related to polycystic ovary syndrome (PCOS). Hum Reprod. 2004; 19(1): 41-47, doi: 10.1093/humrep/deh098, indexed in Pubmed: 14688154.

15. Olszanecka-Glinianowicz M, Madej P, Wdowczyk M, et al. Circulating FGF21 levels are related to nutritional status and metabolic but not hormonal disturbances in polycystic ovary syndrome. Eur J Endocrinol. 2015; 172(2): 173-179, doi: 10.1530/EJE-14-0539, indexed in Pubmed: 25411238

16. Olszanecka-Glinianowicz M, Madej P, Nylec M, et al. Circulating apelin level in relation to nutritional status in polycystic ovary syndrome and its association with metabolic and hormonal disturbances. Clin Endocrinol (Oxf). 2013; 79(2): 238-242, doi: 10.1111/cen.12120, indexed in Pubmed: 23199261.

17. Olszanecka-Glinianowicz M, Madej P, Zdun D, et al. Are plasma levels of visfatin and retinol-binding protein 4 (RBP4) associated with body mass, metabolic and hormonal disturbances in women with polycystic ovary syndrome? Eur J Obstet Gynecol Reprod Biol. 2012; 162(1): 55-61, doi: 10.1016/j.ejogrb.2012.01.026, indexed in Pubmed: 22397743.

18. Olszanecka-Glinianowicz M, Kuglin D, Dąbkowska-Huć A, et al. Serum adiponectin and resistin in relation to insulin resistance and markers of hyperandrogenism in lean and obese women with polycystic ovary syndrome. Eur J Obstet Gynecol Reprod Biol. 2011; 154(1): 51-56, doi: 10.1016/j.ejogrb.2010.08.022, indexed in Pubmed: 20889251.

19. Talmor A, Dunphy B. Female obesity and infertility. Best Pract Res Clin Obstet Gynaecol. 2015; 29(4): 498-506, doi: 10.1016/j.bpobgyn.2014.10.014, indexed in Pubmed: 25619586.

20. de Melo AS, Dias SV, Cavalli Rd, et al. Pathogenesis of polycystic ovary syndrome: multifactorial assessment from the foetal stage to menopause. Reproduction. 2015; 150(1): R11-R24, doi: 10.1530/REP-14-0499, indexed in Pubmed: 25835506.

21. Zak-Golab A, Zahorska-Markiewicz B, Langfort J, et al. [Obesity as inflammatory disease]. Postepy Hig Med Dosw (Online). 2008; 62(4): 249-257, indexed in Pubmed: 18542045

22. Spritzer PM, Lecke SB, Satler F, et al. Adipose tissue dysfunction, adipokines, and low-grade chronic inflammation in polycystic ovary syndrome. Reproduction. 2015; 149(5): R219-R227, doi: 10.1530/REP14-0435, indexed in Pubmed: 25628442.

23. Cai RC, Wei Li, DI JZ, et al. [Expression of omentin in adipose tissues in obese and type 2 diabetic patients]. Zhonghua Yi Xue Za Zhi. 2009; 89(6): 381-384, indexed in Pubmed: 19567114.

24. Olszanecka-Glinianowicz M, Banaś M, Zahorska-Markiewicz B, et al. Is the polycystic ovary syndrome associated with chronic inflammation per se? Eur J Obstet Gynecol Reprod Biol. 2007; 133(2): 197-202, doi: 10.1016/j. ejogrb.2006.10.037, indexed in Pubmed: 17224231.

25. Olszanecka-Glinianowicz M, Zahorska-Markiewicz B, Kocełak P, et al. The effect of weight loss on inflammation in obese women with polycystic ovary syndrome. Endokrynol Pol. 2008; 59(1): 13-17, indexed in Pubmed: 18335395.

26. Watanabe T, Watanabe-Kominato K, TakahashiY, et al. Adipose Tissue-Derived Omentin-1 Function and Regulation. Compr Physiol. 2017; 7(3) 765-781, doi: 10.1002/cphy.c160043, indexed in Pubmed: 28640441. 